\title{
Colonic fibronectin and E-cadherin in mild ulcerative colitis
}

\author{
Sarpdag $\mathrm{F}^{1}$, Yönem $\mathrm{O}^{2}$, Aktas $\mathrm{A}^{3}$, Seckin $\mathrm{Y}^{4}$, Tuncer $\mathrm{E}^{5}$, Ozer $\mathrm{H}^{5}$ \\ Sivas Cumhuriyet University Medical School, Department of Internal Medicine, Sivas, Turkey. \\ ahmetaktas0142@hotmail.com
}

\begin{abstract}
BACKGROUND: Although fibronectin has an important role in wound repair, nearly no human studies to date have investigated its condition in ulcerative colitis (UC) histologically. E-cadherin plays a critical role in the repair of normal epithelial tissues. This study aims to find out the condition of these two molecules in UC. MATERIAL AND METHODS: The records of 22 UC patients during the period of 2004-2009 were retrospectively analyzed. We also included 24 patients with sporadic colorectal cancer (SCC) and 24 patients with normal colonoscopic biopsies who served as the control group. Colonoscopic biopsies were stained with E-cadherin and fibronectin.

RESULTS: The E-cadherin loss was significantly more prominent in the SCC group, followed by the UC group and control group. The situation was reverse for fibronectin. We also observed that while the E-cadherin loss was still ongoing in all of the endoscopically inactive cases, the fibronectin staining resembled the staining pattern of normal individuals in ten out of thirteen UC patients.

CONCLUSION: We suggest that the decrease in E-cadherin, even in the inactive period, might be the cause of why UC is not just a compensatory change in repair of inflammation. The results of staining with fibronectin in UC patients were between normal individuals and SCC patients. Further studies are necessary to confirm our results (Tab. 2, Fig. 6, Ref. 15). Text in PDF www.elis.sk

KEY WORDS: ulcerative colitis, fibronectin, E-cadherin.
\end{abstract}

\section{Introduction}

The major characteristic feature of ulcerative colitis is mucosal tissue damage that needs efficient wound healing. The underlying reason for the persistence of disease symptoms and complications is that the tissue repair is ineffective.

Fibronectin (FN) is a multifunctional extracellular matrix protein that plays an important role in cell adhesion and tissue repair. A variety of physiological functions of FN have also been identified, including cell migration, cell differentiation, cell proliferation and blood coagulation (1). However, despite its important roles during wound healing, our knowledge about its condition in ulcerative colitis patients is limited.

E-cadherin, a calcium-dependent cell-cell adhesion molecule is located in the basolateral membrane along the whole length of the crypt, and it plays a significant role in the development and maintenance of epithelial tissues. In inflamed tissue, the expression of E-cadherin is reduced to ensure the mobility of cells and healing of the ulcer (2). Sommeren et al have suggested that CDH1

\footnotetext{
${ }^{1}$ Internal Medicine Clinic of Numune State Hospital, Sivas, Turkey, ${ }^{2}$ Department of Gastroenterology, Cumhuriyet University, Sivas, Turkey, ${ }^{3}$ Department of Internal Medicine, Cumhuriyet University, Sivas, Turkey, ${ }^{4}$ Department of Gastroenterology, Inonu University, Malatya, Turkey, and ${ }^{5}$ Department of Pathology, Cumhuriyet University, Sivas, Turkey

Address for correspondence: A. Aktas, Dr, Sivas Cumhuriyet University Medical School, Department of Internal Medicine, 58140, Sivas, Turkey Phone: +90.530 .9623735$
}

loci which encode E-cadherin show the susceptibility for UC in a Dutch cohort (3). Whether E-cadherin is de novo decreased in UC patients genetically or it is decreased secondary to inflammation is not clear. Also, the condition of fibronectin in UC has not yet been definitely defined. This article aims to investigate these two questions.

\section{Material and methods}

We conducted a retrospective study using a consecutive series of 22 subjects ( 18 male, 4 female) who were diagnosed with ulcerative colitis during the period from 2004 to 2009 at the Cumhuriyet University Hospital in Sivas, Turkey. We also included 24 patients with sporadic colorectal carcinoma (15 male, 9 female patients) who were diagnosed within the same time period, as well as 24 individuals who were found to have normal findings in colonoscopic biopsy. This study was approved by the Ethics Committee of Cumhuriyet University Faculty of Medicine.

The records of the patients were retrospectively analyzed. Colonoscopy findings, disease severity according to Truelove and Witts criteria, and endoscopic activity according to Rachmilewitz endoscopic activity index were reported. Colonoscopies were performed with GIF Q160 (Olympus, Tokyo, Japan).

\section{Histology}

Biopsy samples embedded in paraffin blocks were cut to a $3-\mu \mathrm{m}$ thickness. Then, the slides were deparaffinized, dehydrated and stained immunohistochemically by avidin-biotin peroxidase 


\section{1-335}

Tab. 1. Main characteristics of the patients with ulcerative colitis.

\begin{tabular}{lc}
\hline Mean age (years) & $40.68 \pm 11.28$ \\
\hline Gender: $\mathrm{n}(\%)$ & $18(81.8 \%)$ \\
$\quad$ Male & $4(18.2 \%)$ \\
Female & $62.18 \pm 35.01$ \\
\hline Mean duration of ulcerative colitis symptoms (months) & \\
\hline Mean duration of ulcerative colitis after the diagnosis (months) & $49.08 \pm 20.02$ \\
\hline Involvement of colon n (\%) & $6(31.8 \%)$ \\
Rectal & $11(50.0 \%)$ \\
Left colon & $4(18.2 \%)$ \\
Extensive & - \\
Pancolitis & \\
\hline Severity of disease according to Truelove and Witts criteria & \\
Mild n (\%) & $15(68.2 \%)$ \\
Intermediate n (\%) & $6(27.3 \%)$ \\
Severe n (\%) & $1(4.5 \%)$ \\
\hline
\end{tabular}

method. Hydrogen peroxide in 3\% concentration (Lab vision, CA) was used as blocking solution, and during the staining procedure, all tissue slides were washed in phosphate-buffered solution (PBS) (Sigma Chemical, St Louis, MO). The slides were incubated with anti-E-cadherin (Neomarkers, Mouse MAb IgG1) and antifibronectin (Neomarkers, Mose Mab-11) primary antibodies for 60 minutes in a closed and moisty staining chamber. After the incubation with primary antibodies, the slides were incubated with secondary antibodies for 60 minutes and then stained with AEC chromogen (Santa-Cruz, CA). The slides were counterstained with Mayer's hematoxylin and covered with aqueous mounting solution.
The staining of both antibodies was evaluated by a semiquantitative method that considers the severity degree of staining. The severity of staining was as follows;

For E-cadherin: Score 0: $>99 \%$ staining, Score 1: 75-99 \% staining, Score 2: $<75 \%$ staining and for fibronectin: Score 0: no staining $(-)$, Score 1: intermediate level of staining $(+)$, Score 2: strong staining $(++)$.

\section{Statistical analysis}

The data were analyzed by using SPSS 14.0 package software. Definitive statistic results were indicated as mean $\pm \mathrm{SD}$, whereas the categorical data were expressed as a number of observations (\%). Chi-square test and Fisher's exact probability test were used for statistical evaluation. A p level of $<0.05$ was accepted as significant.

\section{Results}

We included three groups of patients, namely the ulcerative colitis group, sporadic colorectal cancer group and control group with normal colonoscopic biopsies in the study. There were 22 patients (18 male, 4 female) in the ulcerative colitis group with the mean age of $40.68 \pm 11.28$ years $(20-66)$. The sporadic colorectal cancer group comprised 24 patients ( 15 male, 9 female) with the mean age of $61.66 \pm 12.77$. Our control group was composed of 24 patients with normal colonoscopic biopsies of individuals (13 male, 11 female) with the mean age of $54.00 \pm 14.57$ years (22-78). The main characteristics of the patients with ulcerative colitis are given in Table 1 .

We graded the staining of slides semiquantitatively. Staining scores for E-cadherin are as follows; Score 0: $>99 \%$, score 1: 75-99\%, score $2:<75 \%$, and staining scores for fibronectin are: Score 0: negative staining, Score 1: moderate staining $(+)$, Score 2: intense staining $(++)$ (Fig 1, 2, 3 and 4).

All of the individuals in the control group had an E-cadherin score of zero which means no loss of E-cadherin. In the ulcerative colitis group, 1 patient had a score of zero (4.5\%), while 16 patients

Fig. 1. Score 0 (>99\%) E-cadherin staining in a patient with normal colonic mucosa. A: IHCX25, B: IHCX50.
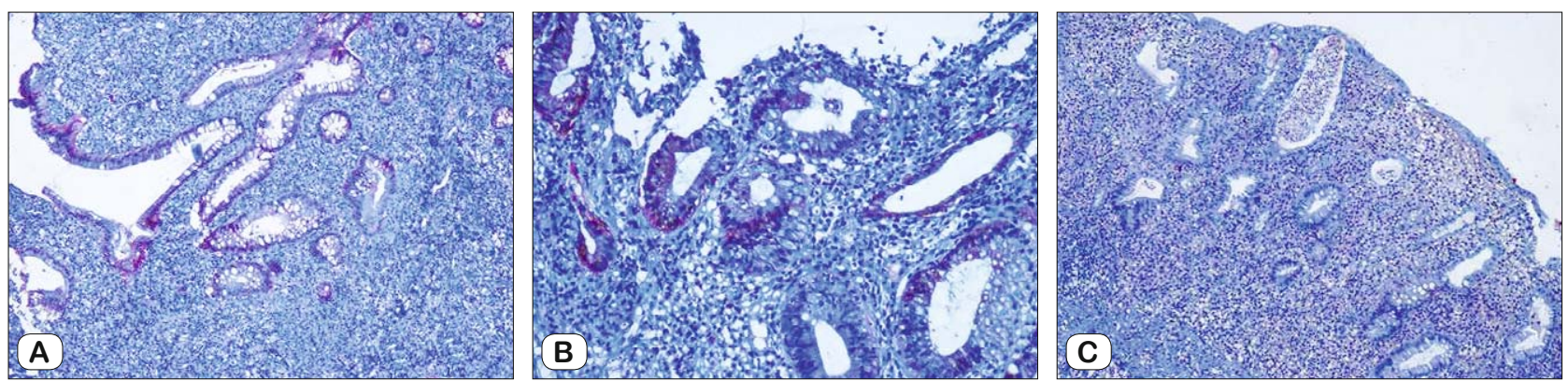

Fig. 2. Score 1 (75-99 \%) E-cadherin staining in a patient with UC. A: IHCX25, B: IHCX50, C: Score $2(<75$ \%) E-cadherin staining in UC patient IHCX25. 
had a score of $1(72.7 \%)$ and five patients had a score of $2(22.7 \%)$. In patients with sporadic colorectal carcinoma, 3 patients had a score of $1(12.5 \%)$ while 21 patients had a score of $2(87.5 \%)$. As a result, we can say that there is no E-cadherin loss in the control group and the loss is statistically more prominent in the sporadic colorectal cancer group than in the ulcerative colitis group. The difference between the three groups as to the E-cadherin staining is statistically significant, while the results for fibronectin differed completely from those for E-cadherin. While no staining of fibronectin was observed in the healthy group, the most intense staining was observed in the colorectal cancer group, followed by the ulcerative colitis group. The distribution of scores is shown in Figures 5 and 6.

We observed that the E-cadherin loss is still ongoing in ulcerative colitis patients who are colonoscopically inactive according to Rachmilewitz endoscopic activity index. There was no difference between the E-cadherin scores in the endoscopically active and inactive periods. We also observed that a high number of colonoscopically inactive patients (10 out of 13) show no staining of fibronectin, which resembles the pattern observed in the control group. However, we could not find any difference in the fibronectin scores in the colonoscopically active and inactive phases of UC (Tab. 2).

\section{Discussion}

E-cadherin is a $120-\mathrm{kDa}$ transmembrane glycoprotein that is localized mainly to the zonula adherens junction, signaling the ex-
Tab. 2. Distribution of E-cadherin and fibronectin staining scores of ulcerative colitis patients according to Rachmilewitz endoscopic activity index.

\begin{tabular}{llccc}
\hline $\begin{array}{l}\text { Stained } \\
\text { antibody }\end{array}$ & $\begin{array}{l}\text { Endoscopic } \\
\text { activity index }\end{array}$ & Score 0 & $\begin{array}{c}\text { Score 1 } \\
\text { and 2 }\end{array}$ & Total \\
\hline E-cadherin (n) & $<4$ (Inactive) & - & 13 & 13 \\
& $>4$ (Active) & 1 & 8 & 9 \\
\hline Fibronectin (n) & $<4$ (Inactive) & 10 & 3 & 13 \\
& $>4$ (Active) & 4 & 5 & 9 \\
\hline
\end{tabular}

$\mathrm{p}=0.41$ for e-cadherin, $\mathrm{p}=0.19$ for fibronectin

tracellular domain cell-cell adhesion through calcium-dependent homotypic interactions (2). Experiments with a chimeric/transgenic murine model indicate the important role of E-cadherin in the maintenance of the intestinal barrier showing that dysregulation of this molecule leads to inflammatory bowel disease (4). In particular, a loss of normal membranous immunoreactivity of Ecadherin and a-catenin is seen in cells surrounding the ulcerated areas in human colonic samples from IBD patients. It is speculated that downregulation of E-cadherin expression could be an important part of epithelial restitution and repair (5). E-cadherin has been found to be downregulated in cells located at the margins of mucosal ulceration during active ulcerative colitis. But the question is whether it is decreased as part of epithelial restitution and repair, i.e., as a compensatory event, or the decrease is genetically associated with the $\mathrm{CDH} 1$ gene locus, and as such it is rather the result, not the cause of ulcerative colitis. Karayiannikis et al investigated E-cadherin's immunoreactivity in colonic tissue specimens of 6 inactive and 6 active ulcerative colitis patients undergoing colectomy and found that E-cadherin expression was preserved in the 6 inactive cases while it was reduced in active cases. We had 13 endoscopically inactive cases without ulcers (having a score lower than 4 according to Rachmilewitz endoscopic activity index) and the E-cadherin loss was observed in all of them, i.e., in none of them it was preserved. Therefore, we suggest that the E-cadherin decrease in UC patients might be the cause of UC not just a compensa-

Fig. 3. Score 1(75-99\%) (A) and Score $2(<75 \%)(B)$ E-cadherin staining in a case with SCC, IHCX25.
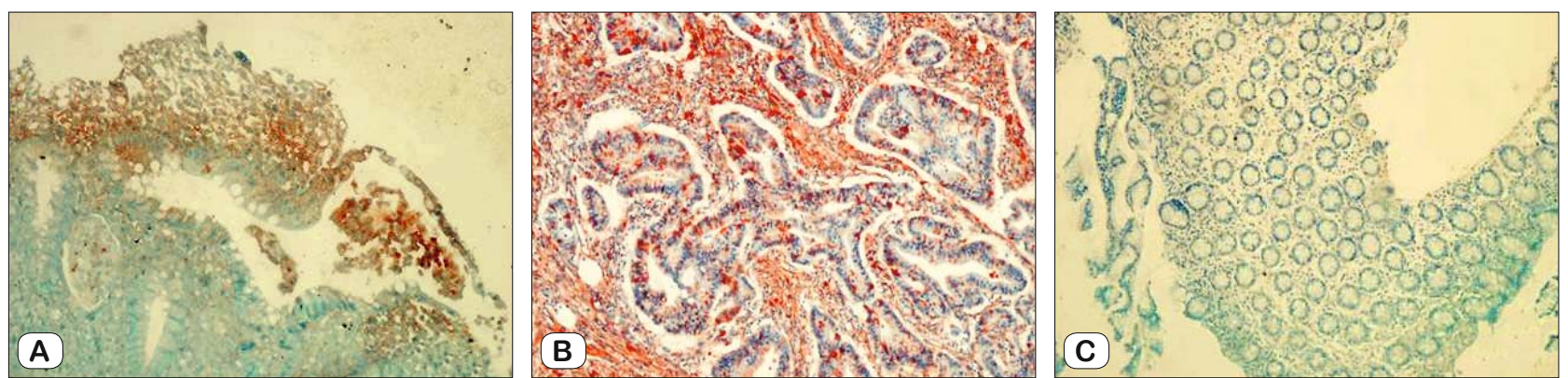

Fig. 4. A: Score 1 [intermediate level of staining $(+)$ ] fibronectin staining in a patient with UC, IHC X25, B: Score 2 [intense staining (++)] fibronectin staining in a patient with SCC, IHCX25, C: Score 0 [no staining $(-)$ ] fibronectin staining in a patient with normal colonic mucosa, IHCX25. 


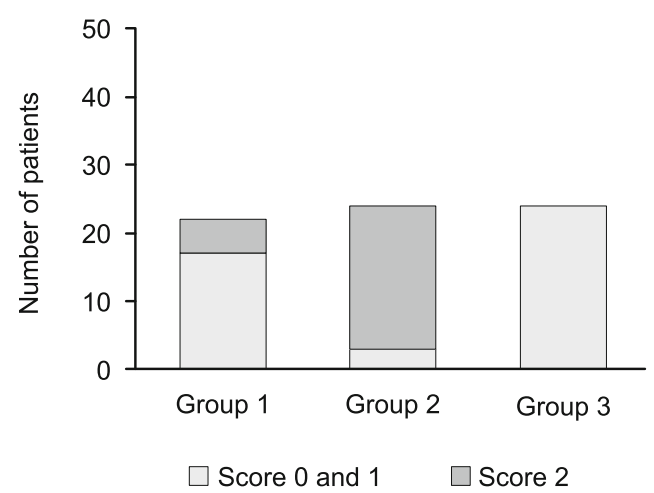

Fig. 5. Distribution of E-cadherin scores within the groups. There was a significant difference between the three groups as to the $\mathbf{E}$-cadherin loss, with no loss in the control group and a most evident loss in the sporadic colorectal cancer group $(p<0.0001)$. Group 1: Ulcerative colitis patients, Group 2: Sporadic colorectal cancer, Group 3: Normal colonoscopic biopsy group; Score 0: > 99 \% staining, Score 1: 75-99 \% staining, Score 2: $<75 \%$ staining.

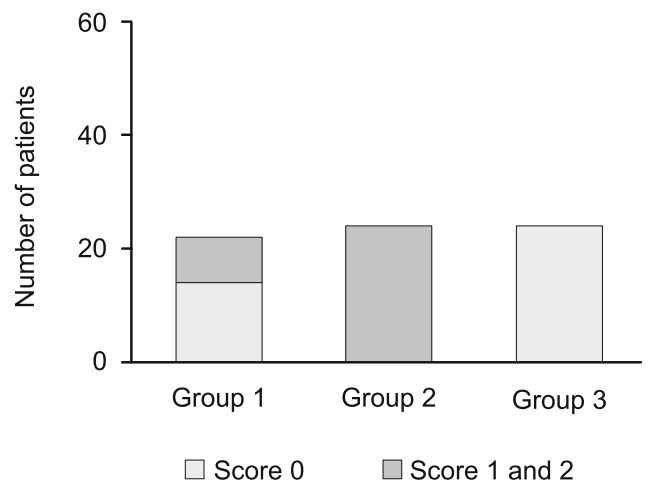

Fig. 6. Distribution of fibronectin scores within the groups. There was a significant difference between the three groups as to the fibronectin staining. While no staining of fibronectin was observed in the healthy group, the most intense staining was observed in the colorectal cancer group $(p<0.0001)$. Group 1: Ulcerative colitis patients, Group 2: Sporadic colorectal cancer, Group 3: Normal colonoscopic biopsy group; Score 0: no staining (-), Score 1: intermediate level of staining $(+)$, Score 2: strong staining $(++)$.

tory change leading to repairment. However, as our number of cases are low, this suggestion needs further studies to be accepted.

$\mathrm{FN}$ is an adhesive glycoprotein with high molecular weight. It is found in the basement membrane, lamina propria, and connective tissue matrices in the intestine (insoluble form) and in body fluids (soluble form). Plasma FN is synthesized predominantly in the liver by hepatocytes and forms a soluble FN dimer as compared with tissue FN, which forms disulfide cross-linked fibrils and is deposited as a matrix (1). Fallinborg et al measured plasma fibronectin concentration in 48 patients with chronic inflammatory bowel disease (CIBD) and in 48 age- and sex-matched healthy controls using electroimmunoassay and a functional (gelatinbinding) assay. They have found out that whereas no difference in immunochemically measured PF was found between the two groups, patients with CIBD had significantly lower gelatin-binding PF than healthy controls (6). Kolachala et al have demonstrated in their mice dextran sodium sulfate-induced colitis model that in normal colon, fibronectin is synthesized and expressed abundantly by the surface epithelial cells; fibronectin synthesis is transcriptionally upregulated in epithelial cells during the acute phase, as well as recovery phase of colitis, and fibronectin modulates the cell attachment through NF-B signaling and binding to integrin receptors (1). In our study, we found that unlike with E-cadherin, the fibronectin staining in endoscopically inactive cases resembles the condition in the normal colon. Therefore, we assume that fibronectin expression in colon reflects inflammation.

Multiple genes contribute to the pathogenesis of UC. CDH1 encodes E-cadherin. CDH1 mutations are known to be associated with multiple epithelial tumors such as gastric cancer, esophageal cancer, and colorectal cancer (7-9). In these tumors, the loss of Ecadherin causes an increase in proliferation, invasion, and metastasis (10-11). Especially the association between CDH1 mutations and colorectal cancer is interesting because patients with $\mathrm{UC}$ are more prone to the development of colorectal cancer $(12,13)$. We investigated three groups, namely the control, ulcerative colitis and sporadic colorectal cancer groups. E-cadherin staining was least observed in the colorectal cancer group, followed by the UC group, and there was no loss in E-cadherin staining in the control group. In our patients, the mean duration of ulcerative colitis after the diagnosis was $49.08 \pm 20.02$ months during which we do not expect the malignity to occur yet and most of our cases had mild activity. Azarschab et al suggested that $\mathrm{CDH} 1$ promoter methylation might be involved in the neoplastic expression from chronic inflammation to colorectal cancer in patients with UC. Notably, promoter methylation of the CDH1 locus has been associated with dysplasia in patients with UC. A potential clinical utility of this finding could lie in identification of patients at high risk of progression to colorectal cancer using promoter methylation as a biomarker (14). Indeed, our study would have been more valuable if we had included a fourth group consisting of UC-induced cancer cases.

Fibronectin has also been implicated in cancer development and growth. Saito et al measured the serum and urinary fibronectin in 113 patients with colorectal cancer and found that fibronectin levels increase with the progression of colorectal cancer. They suggested that fibronectin could be a useful marker of the disease advancement (15). Fibronectin staining was significantly highest in our sporadic colon cancer cases, there was no staining in normal colonic mucosa, and intermediate staining in UC patients. However, our study would be more valuable if we had included UC-induced cancer cases.

\section{Conclusion}

In conclusion we suggest that E-cadherin decrease in U.C patients might be the cause of UC, i.e., not just a compensatory change leading to repairment, and we observed that unlike the expression of E-cadherin, the expression of fibronectin is decreased during the colonoscopically inactive period. We did not include 
UC-induced cancer patients, so we cannot comment on their role during the carcinogenesis period. For our results to be confirmed, further studies with a larger number of patients are needed to be conducted.

\section{References}

1. Wang J, Li R, Li M et al. Fibronectin and colorectal cancer: signaling pathways and clinical implications. J Receptors Signal Transduction 2020; 1-8. doi.org/10.1080/10799893.2020.1817074.

2. Chen S, Song Y. Q, Wang $\mathbf{C}$ et al. Chitosan-modified lipid nanodrug delivery system for the targeted and responsive treatment of ulcerative colitis. Carbohydrate Polymers 2020; 230: 115613. doi.org/10.1016/j. carbpol.2019.115613.

3. Van Sommeren S, Visschedijk MC, Festen EA et al. HNF4 $\alpha$ CDH1 are associated with ulcerative colitis in a Dutch cohort. Inflamm Bowel Dis 2011; 17: 1714-1718. doi.org/10.1002/ibd.21541.

4. Moor Andreas E, Harnik Y, Ben-Moshe S et al. Spatial reconstruction of single enterocytes uncovers broad zonation along the intestinal villus axis. Cell 2018; 175 (4): 1156-1167. e15. doi.org/10.1016/j. cell.2018.08.063.

5. Lopetuso Loris R, De Salvo C, PAstorelli R et al. IL-33 promotes recovery from acute colitis by inducing miR-320 to stimulate epithelial restitution and repair. Proc Nat Acad Sci 2018; 115 (40): E9362-E9370. doi.org/10.1073/pnas.1803613115.

6. Fallingborg J, Nielsen D, Pedersen JO. Decreased gelatin-binding fibronectin in patients with chronic inflammatory bowel diseases. Scand J Gastroenterol 1985; 20: 1062-1064. doi.org/10.3109/00365528509088872.

7. Simões-Correia J, Figueiredo J, Lopes R et al. E-cadherin destabilization accounts for the pathogenicity of missense mutations in hereditary diffuse gastric cancer. PloS one 2012; 7 (3): e33783. doi.org/10.1371/ journal.pone. 0033783 .
8. Liu B, Li X, Li C et al. miR-25 mediates metastasis and epithelialmesenchymal-transition in human esophageal squamous cell carcinoma via regulation of E-cadherin signaling. Bioengineered 2019; 10 (1): 679-688. doi.org/10.1080/21655979.2019.1687391.

9. Song $\mathbf{N}$, Lee J, Cho $\mathrm{S}$ et al. Evaluation of gene-environment interactions for colorectal cancer susceptibility loci using case-only and case-control designs. BMC Cancer 2019; 19 (1): 1231. doi.org/10.1186/s12885-0196456-9.

10. Yu W, Yang $\mathbf{L}, \mathbf{L i} \mathbf{T}$ et al. Cadherin Signaling in Cancer: Function and Therapeutic Target. Front Oncol 2019; 9: 989. doi.org/10.3389/ fonc.2019.00989.

11. Hata K, Anzai H, Ikeuchi H et al. Surveillance colonoscopy for ulcerative colitis-associated colorectal cancer offers better overall survival in real-world surgically resected cases. American J Gastroenterol 2019; 114 (3): 483-489. doi: 10.14309/ajg.0000000000000117.

12. Nadeem MS, Kumar V, Al-Abbasi FA et al. Risk of colorectal cancer in inflammatory bowel diseases. Semin Cancer Biol Acad Press 2020. p. 51-60. doi.org/10.1016/j.semcancer.2019.05.001.

13. Gajendran M, Loganathan P, Jimenez $\mathbf{G}$ et al. A comprehensive review and update on ulcerative colitis. Disease-a-month 2019; 65 (12): 100851. doi.org/10.1016/j.disamonth.2019.02.004.

14. Guz M, Dworzański T, Jeleniewicz W et al. Elevated miRNA Inversely Correlates with E-cadherin Gene Expression in Tissue Biopsies from Crohn Disease Patients in contrast to Ulcerative Colitis Patients. BioMed Research International 2020 2020. doi.org/10.1155/2020/4250329.

15. Saito N, Nishimura H, Kameoka S. Clinical significance of fibronectin expression in colorectal cancer. Mol Med Reports 2008; 1 (1): 77-81. doi.org/10.3892/mmr.1.1.77. 